\title{
Investigación de la Implementación: Características y oportunidades para su práctica.
}

\author{
Implementation Research: characteristics and opportunities for its practice.
}

\author{
Carlos José Fajardo Flores, ${ }^{1}$ Jackeline Alger. ${ }^{2}$
}

${ }^{1}$ Médico General, Maestrante de Epidemiología, Facultad de Ciencias Médicas, UNAH.
${ }^{2} \mathrm{MD}, \mathrm{PhD}$, Unidad de Investigación Científica, Facultad de Ciencias Médica, UNAH.

\begin{abstract}
RESUMEN. El tipo de investigación denominado investigación de la implementación propone aplicar en escenarios reales el conocimiento obtenido a través de medicina basada en evidencia con el objetivo de resolver problemas sanitarios y contribuir a implementar intervenciones efectivas. Se realizó una revisión bibliográfica narrativa a través de una búsqueda avanzada de literatura actualizada utilizando herramientas y plataformas como HINARI, PubMed, Google Académico. La investigación de la implementación puede mediante el uso de protocolos pragmáticos y con metodología mixta, cuantitativa y cualitativa, guiar al personal de salud hacia un nuevo paradigma en investigación. El propósito de la investigación de la implementación es resolver problemas de salud a gran escala, para los que ya existen conocimientos e intervenciones efectivas, pero que por barreras externas no se logran implementar. Este tipo de investigación puede contribuir a resolver muchos de los problemas actuales en el sistema sanitario de Honduras.

Palabras clave: Evaluación de resultado (Atención de salud), Investigación en servicios de salud, Investigación operativa, Proyectos de investigación.
\end{abstract}

\section{INTRODUCCIÓN}

Cada año más de 8 millones de niños mueren por enfermedades prevenibles, entre las cuales podemos mencionar la diarrea, neumonía, malaria, VIH/SIDA, desnutrición, y enfermedades neonatales. ${ }^{1}$ Así mismo, la deficiente rectoría de los sistemas de salud de países de bajos y medianos ingresos genera una carga de morbimortalidad cada vez más insostenible en todos los grupos etáreos. ${ }^{2}$ Sin embargo, existen intervenciones de bajo costo respaldadas por evidencia científica, que están disponibles en cierta manera que podrían prevenir estas muertes, pero que no se han implementado correctamente. ${ }^{1,3}$

En los primeros años de la década anterior muchos reportes de origen anglosajón, señalaban que existía una gran diferencia entre el conocimiento de tratamientos efectivos existentes para muchas enfermedades prevenibles y una pobre implementación de los mismos. Insituciones como United States Department of Health and Human Services, el National Institute of Mental Health (NIMH), Institute of Medicine, entre otros, ${ }^{4,5}$ consideraron que se necesitaba investigación orientada a incrementar la implementación de programas que fueran efectivos y eficientes. Si partimos del hecho que el conocimiento que surge de nuevas investigaciones se hace en ambientes controlados, la realidad a la que se enfrenta este conocimiento hace que no siempre sea aplicable a escenarios reales. ${ }^{6}$ Gracias a este nuevo pensamiento, nace la Investigación de la Implementación

Recibido: 14-5-2018; Aceptado para publicación 13-2-2019

Dirección para correspondencia: Dr. Carlos José Fajardo Flores

Correo electrónico: cjfajardo92@gmail.com

Conflictos de interés. Los autores expresan que no tienen conflictos de interés que declarar en relación a este artículo.

DOI: https://doi.org/10.5377/rmh.v87i2.11917 (implementation research en inglés), que a pesar del progreso mostrado en la última década, aún persisten algunas dificultades para conceptualizar éste término. ${ }^{7}$

En Honduras se generan nuevos conocimientos en materia de salud gracias a los trabajos originales en investigación y las tesis de grado y postgrado cuyas preguntas de investigación han sido inspiradas por problemas de interés nacional. Lamentablemente todo ese conocimiento no llega a ponerse en práctica debido a múltiples barreras incluyendo las relacionadas al talento humano, al paciente, inherentes al sistema de salud, a la falta de recursos y apoyo, entre otras. ${ }^{8}$ Una barrera importante en nuestro país, la cual se suma a las ya descritas es que una proporción importante de estas investigaciones no se publica.

La investigación de la implementación es aún un campo incipiente poco conocido en nuestro medio pero hemos considerado que su metodología resulta apropiada sobremanera para el contexto nacional de salud de Honduras. Nuestro sistema sanitario dispone de poco presupuesto para enfrentar la creciente problemática en salud y de líneas de investigación cuyos protocolos no están diseñados para que sus resultados sean implementados en las comunidades que más lo necesitan. Dentro de las prioridades de investigación de la Secretaría de Salud de Honduras para 2015- 2018, se describe el tema implementación e impacto de políticas, planes y estrategias en salud en la primera línea de investigación (Rectoría y políticas para la salud), ${ }^{9}$ aunque en la práctica no se identificaron ejemplos actuales. Se realizó una revisión bibliográfica narrativa con el objetivo de describir la investigación de la implementación y sus alcances. Se realizó una búsqueda avanzada de literatura actualizada a través de herramientas incluyendo HINARI, PubMed y Google Académico, utilizando los términos implementation re- 
search, operational research, health system research, research designs. Se incluyeron artículos publicados en los últimos 15 años, período de tiempo en el cual se ha propuesto y desarrollado esta área del conocimiento. En el desarrollo de la revisión bibliográfica narrativa se buscó que la mayoría de las referencias fueran de la última década.

\section{ceptos}

Investigación de la implementación: definiciones y con-

La investigación de la implementación es un tipo de investigación científica que estudia los procesos utilizados en la implementación de iniciativas en los sistemas de salud y de los factores contextuales que afectan estos procesos. ${ }^{6} \mathrm{El}$ consenso entre la bibliografía consultada la define como "....el estudio de métodos para mejorar e impulsar, implementar, y trasladar los descubrimientos investigativos a prácticas comunes y rutinarias y reducir el cuidado inapropiado". ${ }^{1,10,11}$ Este concepto relativamente nuevo se enfoca en como promover y apoyar la incorporación y la implementación exitosa de intervenciones basadas en evidencia científica, especialmente las que durante la última

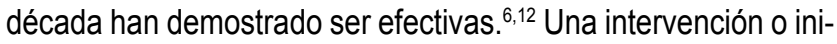
ciativa respaldada por una fuerte evidencia científica, por ejemplo nuevos medicamentos o intervenciones clínicas, pruebas diagnósticas, estrategias de prevención y promoción, y cuya efectividad ha sido comprobada, pueden no tener impacto en la realidad para la que fue propuesta, por el hecho de que todavía existen grandes preguntas sobre cómo se pueden integrar y aplicar efectivamente en el sistema de salud. ${ }^{11,13,14}$

El impacto limitado de las intervenciones, sobre todo en países de bajos ingresos como Honduras, responde a las múltiples barreras (culturales, profesionales, relacionadas con el paciente, recursos, inherentes al sistema, etc.) que se interponen entre la obtención de un conocimiento y realización en el campo de acción. Todos los factores que existen entre estos dos momentos no se han investigado ni abordado., 11,13,15,16 Las características principales de la investigación de la implementación incluyen que debe ser sistemática, multidisciplinaria, contextual y compleja. ${ }^{1}$

\section{Investigación de la implementación en el contexto de escenarios reales}

Existe una brecha enorme entre el conocimiento y la práctica científica. Se estima que los descubrimientos científicos tardan aproximadamente 17 años en convertirse en una práctica rutinaria ya sea clínica o comunitaria. ${ }^{14}$ Constantemente, una gran cantidad de evidencia científica es puesta a disposición de los profesionales en salud y de los tomadores de decisiones. Sin embargo, este valioso conocimiento encuentra diversas barreras a medida que se intenta aplicar al campo real, ya sea este clínico, epidemiológico, salud pública o de rectoría de sistemas de salud. Cochrane et al., ${ }^{8}$ y otros autores ${ }^{3,5,16}$ han reconocido que el primer paso para poder abordar esta situación es identificar y agrupar las barreras de diversas índoles que impiden la implementación y que perpetúan las brechas de teoría-realidad.
Esta diferencia entre lo que sabemos y lo que hacemos, representa un gran reto para los sistemas de salud del mundo y sobre todo en países de bajo ingreso como Honduras, ya que no se aprovechan los avances en el conocimiento y la tecnología disponible, mermando de esta manera los beneficios que el sistema de salud ofrece a la población.7, 7,12

El sistema de salud de Honduras, caracterizado por ser fragmentado y desarticulado, tiene un porcentaje $(8.5 \%)$ de gasto total en salud inferior al promedio de la región de las Américas $(14.1 \%),{ }^{17} \mathrm{y}$ también posee una inversión en área científica y tecnológica de apenas un $0.04 \%$ en relación con el PIB, inferior al nivel regional de América Latina $(0.69 \%) .{ }^{18}$ La evidencia científica fruto de trabajos de investigación originales locales e internacionales, tesis de postgrado, entre otros, no llega a tener un impacto real en nuestro medio. La investigación de la implementación puede ser una herramienta que nos permita trasladar ese conocimiento a la práctica en todas los niveles logrando mayores y mejores resultados con los pocos recursos con los que se cuentan.

\section{Desarrollo de la investigación de la implementación}

Debido a que la investigación de la implementación comprende una amplia variedad de métodos (cualitativos, cuantitativos o mixtos), ${ }^{19}$ resulta inapropiado exponer un solo método que describa o represente este tipo de investigación. Más adecuado sería modificar la forma en que se plantea una pregunta de investigación, pasando de un enfoque menos explicativo a uno más pragmático. Sanders et al., ${ }^{12}$ propone la pregunta: ¿Qué es lo que está pasando en el diseño, implementación, administración, operación o resultados de un programa? $\mathrm{O}$ bien, ¿Es esto lo esperado con esta intervención? ¿Por qué hemos obtenido estos resultados? Es decir, darle ese carácter pragmático a un estudio. Los protocolos de ensayos pragmátivos se diferencian de aquellos que buscan la generación de conocimiento porque los primeros prueban las intervenciones en ambientes controlados. En cambio los pragmáticos buscan maximinar la variabilidad en la forma en que se implementa una intervención, para así poder generalizar los resultados en diferentes escenarios de la vida real. ${ }^{6}$

Hay algunos ejemplos que describen muy bien este concepto. En Sudáfrica, una intervención del sistema de salud llamada STRETCH por sus siglas en Inglés (Streamlining Tasks and Roles to Expand Treatment and Care for HIV), fue creada porque se detectó que la baja cobertura de los pacientes con $\mathrm{VIH}$ que ameritaban tratamiento con antiretrovirales se debía a la poca disponibilidad de personal médico. La estrategia propuso capacitar personal de enfermería para que iniciara y diera seguimiento a pacientes con tratamiento antiretroviral y así elevar la cobertura. Fue a través de un ensayo aleatorizado, con un enfoque pragmático (experimental), que se evaluaron los resultados de dicha estrategia. La evidencia resultante apoyó la estrategia STRETCH y eso se vio reflejado en las políticas nacionales de Sudáfrica a partir de la publicación de los 
resultados..$^{20}$ Otro estudio liderado por Franco Pagnoni, a través del Programa Especial para Investigación y Entrenamiento en Enfermedades Tropicales (TDR), de la OMS, expuso la estrategia "Home Management of Malaria (HMM)" para el tratamiento de la malaria, la cual ha demostrado en poblaciones pequeñas tener un impacto importante en la reducción de la mortalidad y morbilidad por malaria. ${ }^{15,21} \mathrm{~A}$ pesar de la masiva inversión internacional y de los vastos conocimientos de los que se disponen para combatir esta enfermedad, aún se tiene una carga de morbi-mortalidad con niveles intolerables en el África subsahariana. Esta estrategia es el prototipo de la investigación de la implementación, ya que se enfoca en la reducción de esta morbi-mortalidad al incidir en aquellos factores que impiden que el tratamiento para la malaria no complicada llegue a las zonas más inaccesibles. Al mismo tiempo, la estrategia y sus resultados generan nueva información que permite ir cerrando las brechas entre el conocimiento y la realidad en el campo de trabajo. ${ }^{21}$ En estos estudios podemos constatar que sus enfoques pragmáticos logran resolver problemas de salud a gran escala, cuyo principal obstáculo no es la falta de conocimiento sobre una enfermedad, sino aquellas barreras de cualquier índole que no permiten la implementación plena de una estrategia efectiva.

Curran et al., proponen un modelo llamado "Diseño híbrido de efectividad-implementación", ${ }^{22}$ en donde se combinarían los componentes de una investigación clínica y/o epidemiológica, generadora de nuevos conocimientos, con los componentes de diseños metodológicos creados especialmente para que esta nueva información sea implementada adecuadamente. Se espera lograr de esta manera una mayor eficacia y eficiencia de las respuestas en salud que el sistema propone para abordar los problemas que inicialmente suscitaron estas preguntas de investigación. Lawrence et al., realizaron una revisión literaria de investigaciones en servicios de salud mental y analizaron 22 investigaciones publicadas que utilizaron métodos mixtos (cualitativos y cuantitativos), ${ }^{23}$ partiendo de la premisa central que el uso de ambos métodos en combinación pueden proveer un mejor entendimiento del tema investigado que cada uno por separado, dándole más profundidad y mayor amplitud. Sus resultados revelaron que el uso de métodos cuantitativos sirve para medir una intervención y los resultados de su implementación, y los métodos cualitativos ayudan a entender el proceso. El enfoque de la investigación de la implementación, independiente del diseño elegido, debe mejorar la equidad, eficiencia, sostenibilidad, aumento proporcional del uso de una estrategia todo con el fin de mejorar la salud de la población. ${ }^{24}$

\section{Iniciativas para impulsar la Investigación de la Imple- mentación}

La OMS a través del TDR ((Special Program for Research and Training in Tropical Diseases), impulsa una serie de recursos de enseñanza - aprendizaje en el tema de investigación de la implementación (disponible en: http://www.who.int/tdr/capacity/strengthening/ir-training/en/, acceso enero 2019). Una de esas herramientas es el "Implementation Research Toolkit" (diponible en https://www.who.int/tdr/publications/topics/ir-toolkit/ en/, acceso enero 2019), la cual incluye recursos para autoaprendizaje de la investigación de la implementación. El toolkit está diseñado para identificar "cuellos de botella", formulación adecuada de preguntas de investigación, y completar todos los pasos, paso a paso, para formular y ejecutar un proyecto de investigación del tipo de investigación de la implementación. Más recientemente se está promoviendo un curso masivo abierto en línea (MOOC por sus siglas en inglés, massive open online course), que pretende llegar a muchas personas de todo el mundo y sobre todo a regiones con alta carga de morbimortalidad, para que a través de la orientación de expertos se conozcan y adopten las prácticas de la investigación de la implementatación.

El Instituto de la Investigación de la Implementación fundado por National Institutes of Health (NIH) junto con otras instituciones y universidades prestigiosas de Estados Unidos de América, Canadá y Europa, ofrecen un amplio directorio de programas de entrenamiento en la ciencia de la implementación. La finalidad de estos programas es entrenar personal con experiencia en metodología de investigación para que sea capaz de trasladar evidencia científica a la práctica a nivel individual, institucional, comunitaria y política. ${ }^{7,25} \mathrm{~A}$ pesar que la literatura, iniciativas y herramientas sobre la investigación de la implementación se han desarrollado predominantemente en inglés, la investigación de la implementación ha empezado a dar sus primeros pasos en América Latina. De la mano de la OMS, OPS y TDR, "La Alianza para Políticas e Investigación en Sistemas de Salud", impulsa iniciativas para promover la investigación de la implementación en la región. Como parte de esta iniciativa, El Instituto de Efectividad Clínica y Sanitaria (IECS), Buenos Aires, Argentina, brinda mentoría y supervisión a proyectos de investigación de la implementación en distintos aspectos relacionados con la implementación de guías de VIH, Enfermedad de Chagas, sífilis, entre otras. (Información detallada está disponible en https://www.iecs.org.ar/ipier/ acceso enero 2019). La Universidad de Antioquía de Colombia en convenio con OMS y TDR, han puesto en marcha la Maestría en Epidemiología con énfasis en Investigación de la Implementación (http://www. udea.edu.col, acceso enero 2019). Esta Maestría realiza convocatorias y ofrece becas de forma competitiva. En 2018 se realizó la segunda convocatoria. En la primera cohorte de 13 estudiantes que completaron el programa académico 2015-2017, no participó ninguno de Honduras. Igual que en Latinoamérica, bajo el mismo convenio hay otras seis universidades que ofrecen programas de maestria y doctorado en Africa (3), Asia (2) y Oriente Medio (1) (Disponible en http://www.who.int/tdr/capacity/strengthening/postgraduate/en/, acceso enero 2019).

En el marco de todas estas iniciativas, ya sea como programas académicos formales o de herramientas interactivas en internet, podemos ver que la OMS y otras instituciones internacionales han identificado a la investigación de la implementación como una posible solución a la carga creciente de morbimorta- 
lidad en las regiones más pobres del mundo. En la medida en que más profesionales de la salud entren en contacto con este tipo de investigación, más cerca se estará de convertir los más sofisticados logros de la investigación en prácticas rutinarias.

\section{CONCLUSIÓN}

La investigación de la implementación es un tipo de investigación científica con un campo de acción muy amplio, por lo que su utilidad y alcance es ciertamente muy extenso. En este mismo sentido, al definirlo nos encontramos con un concepto general, que puede ser aplicado en muchas disciplinas. No existe un método que represente la investigación de la implementación pero, como pudimos observar en los estudios discutidos, los protocolos que nacen bajo este concepto deben ser pragmáticos y su pregunta de investigación debe responder a preguntas tales como ¿Qué está pasando con la operación de la estrategia, su implementación o administración? ¿Es esto lo que se esperaba? Adicionalmente, debe identificar cuales son aquellas barreras que se interponen entre un conocimiento teórico e impacto en la vida real. Se ha podido observar como una metodología mixta, que genera profundidad en el conocimiento, producto de métodos cuantitativos, puede analizarse simultáneamente a través de métodos cualitativos que ayudan a comprender el proceso, facilitando la implementación de la intervención en cuestión.

En Honduras, es necesario emprender un tipo de investigación que pueda aprovechar los pocos recursos económicos y académicos de los que se disponen. La investigación de la implementación se convierte en una herramienta práctica para guiar el camino de los profesionales clínicos, epidemiólogos, salubristas y los tomadores de decisiones, hacia un nuevo paradigma en investigación, cuyo propósito es resolver problemas de salud a gran escala, para los que ya se cuentan con los conocimientos e intervenciones necesarias, pero que debido a barreras externas no se logran implementar. Es necesario profundizar mucho más en este tema, porque sigue siendo un concepto incipiente y poco conocido, pero que tiene el potencial para revolucionar la manera en que nuestro sistema de salud afronta la inmesa problemática nacional. La disponibilidad en línea de recursos de información y capacitación facilitan la práctica de este tipo de investigación y su incorporación en el sistema de enseñanza e investigación universitario y también de la Secretaria de Salud.

\section{REFERENCIAS}

1. MEASURE Evaluation Implementation Research Technical Working Group. Fundamentals of Implementation Research. [Internet]. Chapel Hill: MEASURE; 2012. [consultado 12 mayo de 2018]. Disponible en: https:// www.measureevaluation.org/resources/publications/ms-12-55

2. Organización Mundial de la Salud. Informe sobre la salud del mundo: sistemas de Salud. [Internet]. Washington D.C.: OMS; 2013.[consultado 23 marzo de 2018]. Disponible en: https://www.who.int/whr/2003/chapter7/es/

3. Larmarange J, Sow K, Broqua C, Akindès F, Bekelynck A, Koné M. Social and implementation research for ending AIDS in Africa. The Lancet Public Health. 2017;2(12):e540.

4. Fixsen D, Naoom S, Blasé K, Friedman R, Wallace F. Implementation Research: A Synthesis of the Literature. Tamps, FL: University of South Florida; 2005

5. Atkins D, Kupersmith J. Implementation Research: A Critical component of realizing the benefits of comparative effectiveness research. Am J Med. 2010;123(12):e38-e45.

6. Peters DH, Tran NT, Taghreed A. Implementation research in health: a practical guide. [Internet]. Geneva: World Health Organization; 2013. [actualizado 20 noviembre de 2013; consultado 1 noviembre de 2017]. Disponible en: https://apps.who.int/iris/handle/10665/91758

7. Sampson UK, Chambers D, Riley W, Glass RI, Engelgau MM, Mensah GA. Implementation Research. Glob Heart. 2016; 11(1):153-158.

8. Cochrane LI, Olson CA, Murray S, Dupuis M, Topman T, Hayes S. Gaps Between Knowing and Doing: understanding and assessing the barriers to Optimal Health Care. J Contin Educ Health Prof. 2007;27(2):94-102.

9. Secretaría de Salud (HN). Agenda de Investigación para la Salud 20152018. Tegucigalpa: Secretaría de Salud ; 2015.

10. Eccles M, Grimshaw J, Walker A, Johnston M, Pitts N. Changing the behavior of healthcare professionals: The use of theory in promoting the uptake of research findings. J Clin Epidemiol. 2005;58(2):107-12.

11. Palinkas $L A$, Saldana $L, C$ hou $C$, Chamberlain $P$. Use of research evidence and implementation of evidence-based practices in youth-serving systems. Child Youth Serv Rev. 2017;83:242-7.

12. Sanders D, Haines A. Implementation Research Is Needed to Achieve International Health Goals. PLoS Med. 2006;3(6):719-22.
13. Remme JHF, Adam T, Becerra-Posada F, D'Arcangues C, Devlin M, Gardner $C$, et al. Defining research to improve health systems. PLoS Med. 2010;7(11):e1001000.

14. Zoellner JM, Porter KJ. Translational Research. In: Coulston AM, Boushey C, Ferruzzi MG, Delahanty LM, editores. Nutrition in the prevention and treatment of disease. London, San Diego CA: Academic Press; 2017. p. $125-43$.

15. World Health Organization, Special Programme for Research and Training in Tropical Diseases. Implementation Research in TDR: conceptual and operational framework. [Internet]. Geneva:who; 2003. [consultado 15 mayo de 2018]. Disponible en: http://www.who.int/tdr/publications/tdrresearch-publications/ide_framework/en/

16. Cidoncha-Moreno MÁ, Rúz de Alegría-Fernandez de Retana B. Percepción de barreras para la utilización de la investigación en enfermeras de Osakidetza. Enferm Clin. 2017;27(5):286-93.

17. World Health Organization. World Health Statistics 2014: Part III, Global Health indicators. [Internet]. Washington D.C.: WHO; 2014. [consultado 15 mayo de 2018]. Disponible en: http://www.who.int/gho/publications/world_ health_statistics/EN_WHS2014_Part3.pdf?ua=1

18. Universidad Nacional Autónoma de Honduras, Dirección de Investigación Científica. Avances de la investigación científica en Honduras. Investigación y Ciencia 2014; 4(2):3-4.

19. Alonge O, Rodriguez DC, Brandes N, Geng E, Reveiz L, Peters DH. How is implementation research applied to advance health in low-income and middle-income countries?. BMJ Glob Health [Internet]. 2019 [consultado 15 mayo de 2018]; 4(2): e001257.

20. Fairall L, Bachmann MO, Lombard C, Timmerman V, Uebel K, Zwarenstein $\mathrm{M}$, et al. Task shifting of antiretroviral treatment from doctors to primarycare nurses in South Africa (STRETCH): a pragmatic, parallel, clusterrandomised trial. The Lancet. 2012 ;380(9845):889-898.

21. Pagnoni F. Malaria treatment: No place like home. Trends Parasitol. 2009;25(3):115-9.

22. Curran G, Bauer M, Mittman B, Pyne J, Stetler C. Effectiveness-implementation hybrid designs: Combining elements of clinical effectiveness and implementation research to enhance public health impact. Med Care. 2012;50(3):217-26. 
23. Palinkas LA, Aarons GA, Horwitz S, Chamberlain P, Hurlburt M, Landsverk J. Mixed method designs in implementation research. Adm Policy Ment Health. 2011;38(1):44-53.

24. Theobald S, Brandes N, Gyapong M, El-Saharty S, Proctor E, Diaz T et al. Implementation research: new imperatives and opportunities in global health. The Lancet. 2018; 392(10160):2214-2228.
25. The VA Center for Implementation Practice and Research Support (ClPRS). Directory of Implementation Science Training Programs.[Internet]. Los Angeles, CA: CIPRS; 2012. [consultado 15 mayo de 2018]. Disponible en: https://obssr.od.nih.gov/wp-content/uploads/2017/03/DI_Training_Directory.pdf.

ABSTRACT. The type of research called implementation research aims to apply in real scenarios the knowledge obtained through evidence-based medicine in order to solve health problems and contribute to the implementation of effective interventions. A narrative bibliographic review was performed through an advanced literature search that was carried out through tools and platforms such as HINARI, PubMed, Google scholar. Implementation research can through pragmatic protocols and with mixed, quantitative and qualitative methodology, guide health personnel to a new paradigm in research. The purpose of implementation research is to solve large-scale health problems, for which the necessary knowledge and effective interventions already exist, but because of external barriers are not managed to implement. This type of research can help to effectively resolve many of the current problems in the health system in Honduras.

Keywords: Health services research, Operations research, Outcome assessment (health care), Research design. 